\title{
Comprehension of Concrete and Abstract Words in Semantic Dementia
}

\author{
Elizabeth Jefferies \\ University of York, UK \\ Roy W. Jones \\ Royal United Hospital, Bath, UK
}

\author{
Karalyn Patterson \\ MRC Cognition and Brain Sciences Unit, UK \\ Matthew A. Lambon Ralph \\ University of Manchester, UK
}

\begin{abstract}
The vast majority of brain-injured patients with semantic impairment have better comprehension of concrete than abstract words. In contrast, several patients with semantic dementia (SD), who show circumscribed atrophy of the anterior temporal lobes bilaterally, have been reported to show reverse imageability effects, that is, relative preservation of abstract knowledge. Although these reports largely concern individual patients, some researchers have recently proposed that superior comprehension of abstract concepts is a characteristic feature of SD. This would imply that the anterior temporal lobes are particularly crucial for processing sensory aspects of semantic knowledge, which are associated with concrete not abstract concepts. However, functional neuroimaging studies of healthy participants do not unequivocally predict reverse imageability effects in SD because the temporal poles sometimes show greater activation for more abstract concepts. The authors examined a case-series of $11 \mathrm{SD}$ patients on a synonym judgment test that orthogonally varied the frequency and imageability of the items. All patients had higher success rates for more imageable as well as more frequent words, suggesting that (1) the anterior temporal lobes underpin semantic knowledge for both concrete and abstract concepts, (2) more imageable items-perhaps because of their richer multimodal representations-are typically more robust in the face of global semantic degradation and (3) reverse imageability effects are not a characteristic feature of SD.
\end{abstract}

Keywords: semantic dementia, imageability, concreteness, anterior temporal, synonym judgment

Supplemental materials: http://dx.doi.org/10.1037/a0015452.supp

How do we represent and process the meanings of concrete and abstract words such as COAT and HOPE? Concrete concepts encapsulate the meanings of tangible things that can be experienced through our senses-consequently, we can readily form mental images for concrete words. Abstract concepts, in contrast, do not refer to physical objects and, for the most part, do not readily evoke mental images: instead these concepts refer to ideas or mental states. In behavioral studies, healthy participants often show faster and more accurate processing for imageable words (DeGroot, 1989; James, 1975; Kroll \& Merves, 1986; Paivio, 1991). Patients with brain-damage normally show an exaggeration of this effect-for example, people with aphasia and deep dyslexia typically make many more errors for abstract than concrete items (Coltheart, 1980; Goodglass et al., 1969; Jefferies et al., 2007).

Elizabeth Jefferies, Department of Psychology, University of York, UK; Karalyn Patterson, MRC Cognition and Brain Sciences Unit, Cambridge, UK; Roy W. Jones, RICE-Research Institute for the Care of Older People, Royal United Hospital, Bath, UK; Matthew A. Lambon Ralph, University of Manchester, UK.

The authors thank the patients and their carers for their generous assistance with this study and John Hodges and Mark Doran for referring some of the patients to us. We would also like to thank Tim Rogers and Paul Hoffman for useful discussions about these data.

The work was supported by a grant from the NIMH (MH64445), an RCUK fellowship awarded to E. Jefferies and an MRC programme grant (G0501632).

Correspondence concerning this article should be addressed to Elizabeth Jefferies, Department of Psychology, University of York, Heslington, York YO10 5DD, United Kingdom. E-mail: ej514@york.ac.uk
However, in a small number of neuropsychological cases, reverse imageability effects have been observed; that is, relative preservation of abstract knowledge (Breedin, Saffran, \& Coslett, 1994; Cipolotti \& Warrington, 1995; Reilly, Grossman, \& McCawley, 2006; Sirigu, Duhamel, \& Poncet, 1991; Warrington, 1975; Yi, Moore, \& Grossman, 2007). Most of the patients showing this pattern have had damage to the anterior temporal lobes (ATL) bilaterally, typically resulting from herpes simplex encephalitis or semantic dementia (SD) (Marshall, Pring, Chiat, \& Robson, 1996, excepted).

This double dissociation suggests that the cognitive and neural organization of concrete and abstract concepts may be partially distinct. Concrete items have sensory referents, whereas abstract items do not (Paivio, 1986). Visual and other sensory processes may therefore contribute to semantic knowledge for concrete concepts, resulting in more semantic features/richer semantic representations for these items (Paivio, 1986; Plaut \& Shallice, 1993). This notion is supported by the fact that people can generate more predicates for imageable words (Jones, 1985). In contrast, abstract concepts might be more dependent on linguistic processes, given that the meaning of these items is strongly affected by sentence context (e.g., Schwanenflugel \& Shoben, 1983).

According to these proposals, reverse imageability effects could result from damage to visual (and possibly other sensory) aspects of semantic knowledge. Consequently, the brain regions damaged in SD might play a particularly important role in visual/sensory knowledge of objects. Patients with SD have relatively circumscribed bilateral atrophy of the anterior and inferior aspects of the ATL, and the extent of this atrophy correlates with the severity of the semantic impairment (Mummery et al., 2000; Nestor, Fryer, \& 
Hodges, 2006). This pattern of brain damage results in a highly specific impairment of semantic memory: other aspects of cognition and language such as phonology, visual processing and decision-making remain largely intact (Hodges, Patterson, Oxbury, \& Funnell, 1992; Snowden, Goulding, \& Neary, 1989). The semantic impairment in SD affects the full range of input and output modalities-including spoken and written words, pictures, real objects, environmental sounds, smells, and touch (Bozeat et al., 2000; Coccia et al., 2004; Luzzi et al., 2007). There is also a significant degree of item-specific consistency when the same items are probed using different semantic tasks (Bozeat et al., 2000; Coughlan \& Warrington, 1981). These findings indicate that the semantic impairment in SD is amodal and not specific to either verbal or nonverbal information (Rogers et al., 2004). The anterior temporal lobes are a plausible substrate for forming amodal semantic representations as they have extensive connections with cortical areas that represent modality-specific information (see also the theory of "convergence zones"; A. R. Damasio, 1989; H. Damasio, Tranel, Grabowski, Adolphs, \& Damasio, 2004; Gloor, 1997). Accordingly, Rogers et al. (2004) implemented a computational model of the ATL semantic system in which semantic representations were formed through the distillation of information required for mappings between different verbal and nonverbal modalities. When the model was damaged, it reproduced the deficits shown by SD patients across different input and output modalities.

Although patients with SD show generalized semantic degradation, there is also evidence to suggest that they have relatively poor knowledge of sensory attributes compared to functional information (though both are markedly impaired). Patients' definitions of pictures and words contain more associative/functional content than sensory/physical information (Lambon Ralph, Graham, Patterson, \& Hodges, 1999; Lambon Ralph, Patterson, Garrard, \& Hodges, 2003; McCarthy \& Warrington, 1988). A similar pattern was found for an individual patient studied by Cardebat, Demonet, Celsis, and Puel (1996) who was unable to draw animals and objects from memory despite producing some functional properties. Moreover, SD patients show poorer definition-to-picture matching when given descriptions that contain sensory rather than functional information (Lambon Ralph et al., 2003). The inferior temporal lobes are thought to underpin the 'ventral visual stream,' which allows object recognition (Ungerleider \& Mishkin, 1982). Given that the focus of atrophy in SD is in anterior, inferior temporal lobes, it is possible that the damaged cortex makes a greater contribution to sensory aspects of semantic knowledge than to functional/associative semantic properties.

If visual/sensory properties are especially vulnerable to damage in $\mathrm{SD}$, we might expect these patients to have more pronounced deficits for imageable than abstract concepts. As noted above, some cases with SD have shown precisely this pattern-that is, reverse imageability effects in semantic tasks (Breedin et al., 1994; Cipolotti \& Warrington, 1995; Papagno, Capasso, Zerboni, \& Miceli, 2007; Reilly, Cross, Troiani, \& Grossman, 2007a; Reilly et al., 2006; Reilly, Peelle, \& Grossman, 2007b; Vesely, Bonner, Reilly, \& Grossman, 2007; Warrington, 1975; Yi et al., 2007). A recent review suggested that better comprehension of abstract than concrete concepts is one of the general features of SD (Grossman $\&$ Ash, 2004). It is important to emphasize, however, that reverse imageability effects have been reported in a relatively small number of studies, which have largely examined single cases. There- fore, it is unclear whether reverse imageability effects are the norm in $\mathrm{SD}$, or whether there is a reporting bias. At least some, though not all, of the reports of patients with reverse imageability effects were accompanied by lesion information implicating the ATLbut this does not establish that ATL lesions predictably produce reverse imageability effects. A recent study did find poorer comprehension of motion verbs compared with cognition verbs in a group of 12 patients with $\mathrm{SD}$, although this effect was not found for nouns in the same definition-to-word matching task (Yi et al., 2007) (see also Reilly et al., 2007a). Moreover, a recent study by Pulvermüller et al. (2008) found poorer performance in a lexicaldecision task for abstract versus concrete words in eight out of 11 SD patients. Crutch and Warrington (2006) also found that comprehension of abstract concepts was impaired in SD although frequency-matched abstract and concrete concepts were not compared. Therefore, additional research is needed to establish if reverse imageability effects are widespread in SD.

Functional neuroimaging studies of neurologically intact participants also provide relevant evidence about the neural organization of concrete and abstract concepts. These studies point to considerable overlap in the network representing abstract/imageable words, although some differences have also been observed. Figure 1 shows sites of peak atrophy and hypometabolism in SD (in yellow) together with peak activations from functional neuroimaging studies that directly contrasted concrete (C) and abstract (A) words. This meta-analysis shows that although individual functional neuroimaging studies might be taken as evidence for the importance of the ATL in concrete or abstract concepts, the pattern across studies is inconsistent. Temporal lobe sites showing greater activation for $\mathrm{C}>\mathrm{A}$ words (in blue/cyan) have almost exclusively been found within occipital, posterior infero-temporal cortex (shown on slices $\mathrm{Y}=-51$ and $\mathrm{Y}=-41)$ and medial ATL sites $(\mathrm{Y}=-21 ; \mathrm{Y}=$ -11 , including one peak in left inferior temporal pole (slice $\mathrm{Y}=$ 19) (Fiebach \& Friederici, 2003; Noppeney \& Price, 2002; Sabsevitz, Medler, Seidenberg, \& Binder, 2005; Whatmough, Verret, Fung, \& Chertkow, 2004; Wise et al., 2000). Meanwhile, sites showing greater activation for $\mathrm{A}>\mathrm{C}$ words (in red/pink) occurred in more diverse areas linked to language processing, especially left posterior superior temporal areas (including the superior parts of the temporal poles bilaterally; shown on slices $\mathrm{Y}=9$ and $\mathrm{Y}=19$ ) and left inferior frontal gyrus ( $\mathrm{Y}=9, \mathrm{Y}=19$ ) (Binder et al., 2005; Kiehl et al., 1999; Noppeney \& Price, 2004; Perani et al., 1999; Sabsevitz et al., 2005; Whatmough et al., 2004). These patterns are broadly consistent with the proposal that concrete concepts are more reliant on occipital-temporal areas that underpin visual object recognition (Ungerleider \& Mishkin, 1982), whereas abstract concepts depend more on brain regions responsible for verbal comprehension (e.g., Scott, Blank, Rosen, \& Wise, 2000). However, the functional neuroimaging findings do not unequivocally predict reverse imageability effects in SD. As revealed by Figure 1, SD patients show atrophy and hypometabolism across the ATL, affecting both superior temporal pole areas that might be particularly critical for abstract words and medial ATL regions that might play a greater role in processing concrete words. Moreover, there is considerable overlap in the areas activated by $\mathrm{C}$ and $\mathrm{A}$ concepts in the ATL. Of the 12 studies reviewed here (see Figure 1 for details), ATL activation $(\mathrm{Y}>-4)$ was observed in five studies for $\mathrm{A}>\mathrm{C}$ and two studies for $\mathrm{C}>\mathrm{A}$, suggesting a high level of inconsistency and substantial numbers of null results. From this, we would 


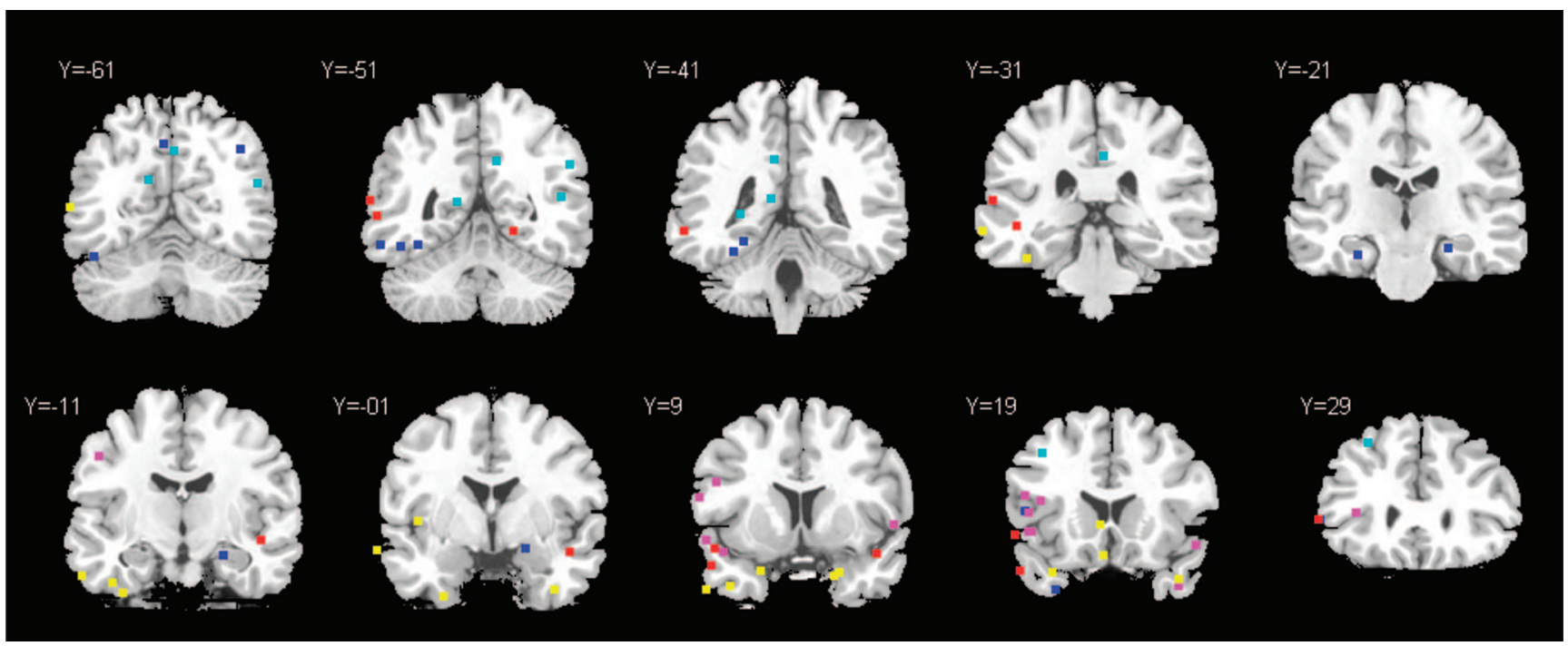

Figure 1. Meta-analysis of functional neuroimaging studies directly comparing concrete and abstract words. Note: Twelve functional neuroimaging studies supplied the peaks (Binder et al., 2005; Fiebach \& Friederici, 2003; Giesbrecht, Camblin, \& Swaab, 2004; Grossman et al., 2002; Jessen et al., 2000; Kiehl et al., 1999; Noppeney \& Price, 2002, 2004; Perani et al., 1999; Sabsevitz et al., 2005; Whatmough et al., 2004; Wise et al., 2000). Red/Pink $=$ sites showing greater activation for abstract stimuli (pink = lexical decision; red $=$ other tasks, primarily semantic judgments). Blue/cyan = sites showing greater activation for concrete stimuli $($ cyan $=$ lexical decision; blue $=$ other tasks, primarily semantic judgments). Yellow $=$ sites of peak atrophy (Mummery et al., 2000) and hypometabolism (Nestor et al., 2006) in SD.

expect the overwhelming majority of SD patients to show substantial deficits for both concrete and abstract items. Furthermore, given that normal language users tend to reveal a $\mathrm{C}>\mathrm{A}$ advantage in many tasks, we predict that, as comprehension deteriorates in $\mathrm{SD}$, it will largely maintain this $\mathrm{C}>\mathrm{A}$ profile. On this account, the reported SD cases showing $\mathrm{A}>\mathrm{C}$ would be occasional deviations from the typical pattern.

The current study examined this issue by assessing the comprehension of concrete and abstract words in a case-series of 11 patients. Our sample should be unaffected by the "reporting bias" discussed above (i.e., the tendency to selectively publish case reports that show reverse concreteness effects), because the patients were selected only on the basis that they had a diagnosis of SD and were available for testing. The patients ranged in severity from mild to severe, allowing an investigation of the relationship between the degree of semantic impairment and the size of any difference between abstract and concrete words. More severely impaired cases might be less likely to reveal a difference in either direction because the atrophy in SD spreads as the disease progresses. We used a synonym judgment task that orthogonally varied the frequency and imageability of the items. Word frequency was manipulated as well as imageability for two reasons. First, this allowed the test to be sensitive to imageability effects in both mild and severely impaired cases (avoiding floor and ceiling effects). Second, the frequency findings are of interest in their own right. Although the meanings of frequently encountered words/pictures are reported to be better preserved than less frequent stimuli in SD, this work is limited to picture naming (Lambon Ralph, Graham, Ellis, \& Hodges, 1998), regression analyses of comprehension tasks (Bozeat et al., 2000) and single case-studies (Funnell, 1995).
As well as addressing these theoretical considerations, this paper also has a practical motivation: we publish a new synonym test that has some advantages over the existing alternatives. (1) The test examines the influence of imageability and frequency at the same time. These factors are varied orthogonally so that interactions between them can be investigated. (2) The test includes substantial variation of both of these variables. (3) Frequency and imageability are manipulated for the response choices as well as for the probe words, increasing the sensitivity of the test to these effects. (4) There are three rather than the usual two response choices per trial, reducing the chance rate from 0.5 to 0.33 .

\section{Method}

\section{Test Construction}

There were 96 trials split evenly between two frequency bands [mean frequency of probe words (with standard deviations in parentheses) $=128(102)$ and $4.6(4.5)$ counts per million in the Celex database; Baayen, Piepenbrock, \& van Rijn, 1993] and three imageability bands [mean imageability of probe words $=275$ (17.3), 452 (26.0) and 622 (14.0) respectively, on a scale of 100700, from the MRC Psycholinguistic Database; Coltheart, 1981]. The frequency ranges of the high/low frequency sets did not overlap, and similarly, the high, medium, and low imageability words had nonoverlapping imageabilities. Frequency and imageability were varied orthogonally; there were 16 trials in each of the six frequency-byimageability conditions. Frequency was matched in triplets across the high, medium, and low imageability words, and imageability was matched pairwise for the high and low frequency sets. Target words (i.e., the intended correct choice) were presented alongside two un- 
related distracters. Both the targets and distracters were matched to the probe word for frequency and imageability. As a consequence, imageability and frequency were varied in the trial as a whole. The conditions were not matched for word length (average $=5.6,6.5$ and 7.7 letters per word for the high, medium, and low imageability conditions, respectively). Simultaneous auditory and visual presentation was used and patients indicated their choice by pointing. The test was not timed. The items are provided in supplemental material published online.

\section{Participants}

The synonym judgment task was administered to 11 patients with a clinical diagnosis of SD, recruited from Cambridge, Bath or Liverpool, U.K. IRB approval was provided by a Multi-Centre Research Ethics Committee (covering patients in Bath and Liverpool) and the Cambridgeshire Research Ethics Committee. The patients fulfilled all of the published criteria for SD (e.g., Hodges et al., 1992): they had word-finding difficulties in the context of fluent speech and showed impaired semantic knowledge and single word comprehension; in contrast, phonology, syntax, visual-spatial abilities, and day-to-day memory (assessed informally in conversation) were relatively well preserved. Table 1 provides demographic details and background neuropsychological scores on tasks administered periodically as part of our standard battery of assessments. All of the scores were obtained within a year of the synonym judgment task. Magnetic resonance imaging revealed focal atrophy of the anterior temporal lobes bilaterally in every case.

Eleven healthy participants matched in age to the SD group (age range 56-65) also completed the synonym judgment task. They had an average of 15.3 years of education. There was no relation- ship between educational level and synonym judgment performance (for either the controls or the patients). All of the participants (patients and controls) provided written consent.

\section{Results}

The results of the synonym judgment test are shown in Figure 2. The SD patients performed substantially more poorly than controls in every condition, $t(20)=9.6-2.3, p<.04$; Cohen's $d=1.2-4.2$. The control group showed a positive effect of higher imageability $(F(2$, $20)=7.2, p=.004$; partial Eta squared $\left.\left(\eta_{\mathrm{p}}^{2}\right)=.42\right)$ although performance was near ceiling on all conditions (there was no main effect of frequency and no interaction). The SD patients' comprehension showed strong positive effects of both higher imageability $(F(2$, $\left.20)=25.3, p<.0001 ; \eta_{\mathrm{p}}^{2}=.72\right)$ and higher frequency $(F(1$, $\left.10)=62.6, p<.0001 ; \eta_{\mathrm{p}}^{2}=.86\right)$. The interaction between these factors approached significance $\left(F(2,20)=2.9, p=.08 ; \eta_{\mathrm{p}}^{2}=.22\right)$. For high frequency items, medium imageability words were understood better than low imageability words (Bonferroni $t(10)=4.7, p=$ $.004)$ but there was no advantage for high over medium imageability words $(t(10)<1)$. For low frequency items, accuracy was significantly greater for high versus medium imageability words (Bonferroni $t(10)=4.0, p=.01)$ but the difference between medium and low imageability words did not reach significance $(t(10)=1.9, n s)$. These patterns of significance can be interpreted as follows: (1) Frequent words are understood comparatively well by patients with SD so their performance was only affected by the other variable - imageabilitywhen it reached its lowest value. (2) SD patients are so poor at comprehending low-frequency words that their performance had already dropped to a low level for medium-imageability words; it

Table 1

Biographical Details and Background Neuropsychological Scores

\begin{tabular}{|c|c|c|c|c|c|c|c|c|c|c|c|c|}
\hline & $\mathrm{AN}$ & LS & SJ & WM & EK & $\mathrm{ATe}$ & GE & $\mathrm{KI}$ & GT & $\mathrm{PD}$ & MK & No. items \\
\hline Age & 64 & 60 & 59 & 54 & 59 & 66 & 50 & 65 & 70 & 72 & 67 & \\
\hline Sex & M & M & $\mathrm{F}$ & $\mathrm{F}$ & $\mathrm{F}$ & M & $\mathrm{M}$ & M & M & $\mathrm{F}$ & $\mathrm{F}$ & \\
\hline Education (leaving age) & 14 & 18 & 16 & 21 & 15 & 24 & 16 & 14 & 14 & 14 & 17 & \\
\hline Composite semantic score & 1.7 & 1.2 & 0.8 & 0.6 & 0 & -0.2 & -0.4 & -0.5 & -0.5 & -1.4 & -1.4 & \\
\hline Word-picture match & 63 & 63 & 59 & 52 & 46 & 58 & 32 & 36 & 32 & 17 & 11 & 64 \\
\hline Picture naming & 53 & 43 & 29 & 26 & 17 & 10 & 13 & 15 & 11 & 4 & 2 & 64 \\
\hline PPT: Pictures & NT & 49 & 48 & 44 & 35 & 47 & 34 & 31 & 37 & 26 & 33 & 52 \\
\hline PPT: Words & NT & 49 & 42 & 39 & 36 & 44 & 28 & 35 & 32 & 26 & 26 & 52 \\
\hline CCT: Pictures & 49 & 53 & 51 & 52 & 33 & 40 & 32 & 20 & 27 & 17 & 26 & 64 \\
\hline CCT: Words & 55 & 54 & 47 & 30 & 26 & 45 & 27 & 33 & 28 & 24 & NT & 64 \\
\hline Fluency: 8 categories & 60 & 32 & 31 & 30 & 31 & 6 & 22 & 27 & 24 & 7 & 1 & - \\
\hline Fluency: 3 letters & 32 & 7 & 23 & NT & 29 & 8 & 19 & 17 & 24 & 22 & 2 & - \\
\hline Raven's Matrices & 35 & 31 & 34 & 35 & 33 & 32 & 33 & 21 & 35 & 25 & 22 & 36 \\
\hline Digit span forwards & 8 & 8 & 5 & 8 & 6 & 7 & 7 & 8 & 6 & 7 & 5 & - \\
\hline Digit span backward & 6 & 7 & 3 & 5 & 7 & 4 & 4 & 5 & 4 & 5 & 4 & - \\
\hline Rey figure copy & 36 & 21 & 33 & 36 & 34 & 36 & 35 & 34 & 34 & 36 & 30 & 36 \\
\hline VOSP: Screening & NT & 16 & 20 & NT & 20 & 19 & 20 & 20 & 20 & 19 & 17 & 20 \\
\hline VOSP: Dot counting & NT & 10 & 10 & NT & 10 & 10 & 10 & 10 & 10 & 10 & 10 & 10 \\
\hline VOSP: Position discrimin & 20 & 20 & 20 & NT & 20 & 20 & 20 & 19 & 20 & 16 & 17 & 20 \\
\hline VOSP: Number location & 10 & 10 & 10 & NT & 10 & 8 & 9 & 10 & 10 & 9 & 6 & 10 \\
\hline
\end{tabular}

Note. Patients are arranged in order of composite semantic score, derived from word-picture matching, picture naming, picture CCT, and category fluency. PPT $=$ Pyramids and Palm Trees test (Howard \& Patterson, 1992); CCT = Camel and Cactus Test, a test of semantic associations for words and pictures, similar to the PPT test but with four choices per trial (Bozeat et al., 2000); Fluency = total number of words produced in 1 minute, from eight semantic categories and three letters combined. Raven's Matrices = Coloured Progressive Matrices Test, tapping nonverbal reasoning (Raven, 1962); Digit span from Wechsler Memory Scale (Wechsler, 1987); Rey Figure copy involved copying a complex geometrical figure. VOSP = Visual Object and Space Perception battery (Warrington \& James, 1991). 


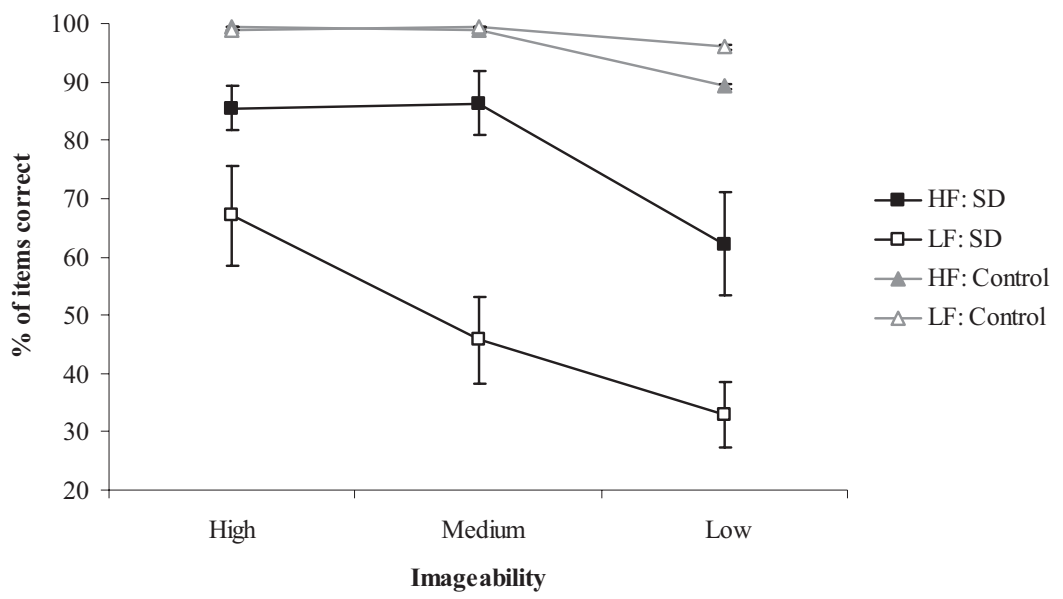

Figure 2. Performance on the synonym judgment task. Error bars show SEM.

declined further (essentially to chance) for the lowest value of imageability, but there was no room for this decrease to reach statistical significance.

Every individual SD patient showed better comprehension of high than low frequency words (Fisher exact one-tailed $p=.06$ to $<.0001$; Cramer's $V=.18$-.64; see Table 2). The majority of the SD group also demonstrated significantly better comprehension of the more imageable words (9/11 patients; Fisher exact two-tailed $p=.05$ to $<.0001$; Cramer's $V=.24-.47$ ). Two cases, GE and KI, who did not show significant positive effects of imageability were investigated in more detail. GE's performance was at ceiling for high frequency words and at floor for low frequency words, regardless of imageability. He was therefore tested on an additional set of medium frequency items to avoid floor and ceiling effects. On these items there was a significant positive effect of imageability (high imageability $=15 / 16$ correct; medium imageability $=11 / 16$; low imageability $=7 / 16$; Fisher's exact two-tailed $p=.01$; Cramer's $V=.44$ ). The other exception was patient KI, who showed only a nonsignificant trend toward better performance for more imageable items on the original test. When KI was retested approximately a month later, his performance revealed a clear imageability advantage (high imageability $=27 / 32$ correct; medium imageability $=19 / 32$; low imageability $=14 / 32$ collapsing across frequency; Fisher's exact two-tailed $p=.003$ ). The imageability effect remained significant when the data from the two test sessions were combined ( $p=.02$; Cramer's $V=.20$ ). We can therefore conclude that all 11 patients showed better comprehension of more imageable words. Reverse imageability effects were absent from the group.

To examine whether comprehension differences between abstract and concrete words varied with the severity of SD, a composite semantic score was derived from the background semantic tests available for all cases (picture naming, word-picture matching, and Pyramids and Palm Trees test for pictures and category fluency; see Table 1). This showed a significant positive correlation with overall synonym judgment performance $(r=.66$, onetailed $p=.01$ ) and with five of the six individual frequency/ imageability conditions ( $r=.54$ to .76 , one-tailed $p<.05$ ); the one exception was the high frequency, high imageability condition that was prone to ceiling effects. There was no relationship between the severity of the semantic impairment and the size of frequency/imageability effects (as measured by the difference be-

Table 2

Synonym Judgement Results for Individual SD Patients

\begin{tabular}{|c|c|c|c|c|c|c|c|c|c|c|c|}
\hline & AN & LS & SJ & WM & EK & $\mathrm{ATe}$ & GE & KI & GT & PD & MK \\
\hline HI, HF & 93.8 & 62.5 & 100 & 100 & 93.8 & 75.0 & 93.8 & 90.6 & 81.3 & 75.0 & 75.0 \\
\hline HI, LF & 100 & 87.5 & 100 & 93.8 & 87.5 & 18.8 & 43.8 & 56.3 & 62.5 & 56.3 & 31.3 \\
\hline MI, HF & 100 & 93.8 & 100 & 100 & 100 & 50.0 & 100 & 75.0 & 87.5 & 87.5 & 56.3 \\
\hline MI, LF & 56.3 & 62.5 & 87.5 & 62.5 & 43.8 & 12.5 & 37.5 & 34.4 & 43.8 & 62.5 & 0 \\
\hline LI, HF & 93.8 & 68.8 & 81.3 & 93.8 & 62.5 & 6.3 & 93.8 & 65.6 & 43.8 & 56.3 & 18.8 \\
\hline LI, LF & 62.5 & 25.0 & 43.8 & 56.3 & 37.5 & 0 & 25.0 & 37.5 & 37.5 & 31.3 & 6.3 \\
\hline HF average & 95.8 & 75.0 & 93.8 & 97.9 & 85.4 & 43.8 & 95.8 & 77.1 & 70.8 & 72.9 & 50.0 \\
\hline LF average & 72.9 & 58.3 & 77.1 & 70.8 & 56.3 & 10.4 & 35.4 & 42.7 & 47.9 & 50.0 & 12.5 \\
\hline $\mathrm{HI}$ average & 96.9 & 75.0 & 100 & 96.9 & 90.6 & 46.9 & 68.8 & 73.4 & 71.9 & 65.6 & 53.1 \\
\hline MI average & 78.1 & 78.1 & 93.8 & 81.3 & 71.9 & 31.3 & 68.8 & 54.7 & 65.6 & 75.0 & 28.1 \\
\hline LI average & 78.1 & 46.9 & 62.5 & 75.0 & 50.0 & 3.1 & 59.4 & 51.6 & 40.6 & 43.8 & 12.5 \\
\hline Total correct & 84.4 & 66.7 & 85.4 & 84.4 & 70.8 & 27.1 & 65.6 & 59.9 & 59.4 & 61.5 & 31.3 \\
\hline
\end{tabular}

Note. Table shows percentage of items that were correct in each condition. Patients are arranged in order of composite semantic score. HI $=$ high imageability; $\mathrm{MI}=$ medium imageability; $\mathrm{LI}=$ low imageability; $\mathrm{HF}=$ high frequency; $\mathrm{LF}=$ low frequency. 
tween these conditions). In addition, there was no correlation between educational level and any aspect of synonym judgment performance in SD-including accuracy in each condition and the magnitude of frequency/imageability effects.

\section{Discussion}

This study examined the impact of word frequency and imageability on synonym judgment in a case-series of 11 patients with SD. Every case showed significantly better comprehension of high than low imageability words, along with more intact understanding of high compared with low frequency words. We did not observe a single instance of the reverse imageability effect reported previously for a few individual SD patients. In addition, there was no relationship between the degree of semantic impairment and the size of the imageability effect. Although the majority of patients showing relative preservation of abstract concepts to date have had damage to the ATL bilaterally, either in the context of SD or herpes simplex encephalitis, these studies have mostly examined single cases that were presumably selected because of the interesting nature of their semantic impairment. Investigations of single cases cannot resolve the question of whether ATL lesions consistently produce reverse imageability effects. Our case-series study of 11 patients indicates that, contrary to the suggestion that reverse imageability effects may be the norm in SD (Grossman \& Ash, 2004), the typical pattern in SD is an advantage for concrete or high-imageability concepts. The impression of an association between SD and reverse imageability effects in the literature is likely to result from a reporting bias.

The marked frequency effect observed here fits with all known research on $\mathrm{SD}$; it is mainly noteworthy because it is perhaps the clearest demonstration so far of the impact of this variable on performance in a receptive task, rather than the expressive tasks (such as object naming, reading, past-tense verb generation, etc.) in which frequency effects have been amply documented in SD (e.g., Funnell, 1995; Lambon Ralph et al., 1998; Patterson et al., 2006; Woollams et al., 2007). More frequently encountered items are thought to form stronger semantic representations than less frequent ones, making them less vulnerable to degradation in SD (Rogers \& McClelland, 2004). Other factors might also contribute to this effect, however; frequent concepts are typically acquired at an earlier age and continue to be encountered regularly as the semantic system degrades. This continued exposure may also afford them some protection from degradation in SD (see Lambon Ralph et al., 1998).

The marked positive imageability effect is more newsworthy because it clearly contradicts previous suggestions that reverse effects of imageability are the norm in this group. Our findings are inconsistent with the idea that the ATL is strongly specialized for visual aspects of knowledge. Instead, this brain region appears to underpin a single semantic store that is critical for understanding all types of stimuli, both concrete and abstract. Abstract words might have fewer semantic features and/or more impoverished semantic representations than imageable words (Paivio, 1986; Plaut \& Shallice, 1993). Healthy participants are able to generate more predicates for imageable words, suggesting that these items have richer semantic representations (Jones, 1985). Even the control participants in this study revealed some benefit in choosing synonyms for high-imageability words. The outcome for the SD patients was therefore just an extension of the normal pattern. If the idea of richer, more detailed representations for concrete concepts is plausible, then it is also plausible that-as semantic memory deteriorates - the amount of information necessary to perform the forced-choice synonym judgment task will drop below "threshold" sooner for abstract than for concrete concepts.

Our finding that SD patients were impaired at both concrete and abstract concepts is broadly consistent with neuroimaging studies that have found overlapping activation for these items within the ATL. It is important to note, however, that patient and neuroimaging studies provide rather different information about the neural basis of conceptual knowledge. The current neuropsychological investigation suggests that the ATL plays a critical role in abstract as well as concrete knowledge; however, we cannot rule out the possibility of functionally dissociable regions within this region (e.g., medial vs. superior ATL for concrete and abstract concepts respectively). Moreover, functional neuroimaging studies show widespread and partially distinct areas of brain activation for concrete and abstract items beyond the ATL, indicating that the wider neural networks that support these functions may be different. Transcranial magnetic stimulation (TMS) in healthy volunteers may provide a means of establishing which specific areas (1) within ATL and (2) beyond ATL are critical for understanding abstract and concrete concepts (Pobric, Lambon Ralph, \& Jefferies, in press).

Given our findings, how is one to understand the published reports of an abstractness advantage in a few patients with ATL lesions? There are at least two possibilities. First, although the temporal pole forms amodal representations of concepts by interacting with modality-specific areas devoted to sights, sounds, words, smells, touch, and so forth, there might be some specialization in areas of the temporal lobe as these inputs come together. The meta-analysis of neuroimaging studies shown in Figure 1 partially supports this view. There are peak activations for the abstract $>$ concrete contrast all the way along the superior aspects of the temporal lobes. Previous research has shown that superior temporal cortex underpins speech comprehension, with more anterior areas responding only to intelligible speech and posterior areas uninfluenced by intelligibility (Crinion et al., 2003; Davis \& Johnsrude, 2003; Narain et al., 2003; Scott et al., 2000). Superior temporal cortex might show greater activation for abstract words, at least in some studies, because these stimuli are highly reliant on this verbal comprehension pathway. In contrast, temporal lobe peaks for the concrete $>$ abstract comparison fall within inferior and medial temporal cortex, among other areas. Similar regions are activated by visual object recognition (Kellenbach, Hovius, \& Patterson, 2005; Stewart, Meyer, Frith, \& Rothwell, 2001), mental imagery (D'Esposito et al., 1997) and picture-based semantic tasks (Adams \& Janata, 2002; Bright, Moss, \& Tyler, 2004; Vandenberghe, Price, Wise, Josephs, \& Frackowiak, 1996). One possibility, therefore, is that reverse concreteness effects occur in patients with an unusual distribution of ATL atrophy-for example, in cases with relative sparing of superior aspects of the ATL despite pronounced damage to medial temporal structures, or following the spread of atrophy to more posterior areas of inferior temporal cortex (especially in cases with only a mild degree of ATL atrophy). Further comparative studies of patients with different distributions of temporal lobe damage are required to test this atrophydistribution hypothesis.

A second possibility is that individual differences in education, interests, and experiences may substantially change the relative fre- 
quency with which concrete and abstract words are encountered and produced by patients premorbidly and/or during the course of the disease. At least some of the SD cases who have shown reverse concreteness effects have been highly educated; for example, patient DM studied by Breedin et al. (1994) was a professional with a master's degree and patient AB examined by Warrington (1975) was a high-ranking civil servant. These individuals might have had greater familiarity with less frequent abstract words, protecting these concepts to some degree from the effects of semantic degradation. Against this hypothesis, there was no clear relationship between educational level and the size of the imageability effect in the current study. We have demonstrated that high levels of education are not always accompanied by reverse concreteness effects (one of the patients showing the standard pattern here had obtained a $\mathrm{PhD}$ ). Nevertheless, the number of years spent in education is at best a crude measure of individual differences in premorbid exposure to abstract and concrete vocabulary. In addition, continued use of abstract and concrete words later in life might be a more critical factor. Very little is currently known about the fate of general versus specialized, expert knowledge in $\mathrm{SD}$; therefore, an interesting question for further research is the extent to individual differences in education or ongoing experiences affect the profile of semantic degradation across different categories of knowledge.

The main contribution of the current study is to show that these individual cases with reverse imageability effects are not representative of SD more generally. Instead, every patient in our case-series study showed poorer comprehension of abstract than concrete words, suggesting that the ATL semantic system underpins the meanings of both imageable and abstract concepts.

\section{References}

Adams, R. B., \& Janata, P. (2002). A comparison of neural circuits underlying auditory and visual object categorization. NeuroImage, 16, 361-377.

Baayen, R. H., Piepenbrock, R., \& van Rijn, H. (1993). The CELEX Lexical Database [CD-ROM]. Philadelphia: Linguistic Data Consortium, University of Pennsylvania.

Binder, J. R., Westbury, C. F., McKiernan, K. A., Possing, E. T., \& Medler, D. A. (2005). Distinct brain systems for processing concrete and abstract concepts. Journal of Cognitive Neuroscience, 17, 905-917.

Bozeat, S., Lambon Ralph, M. A., Patterson, K., Garrard, P., \& Hodges, J. R. (2000). Non-verbal semantic impairment in semantic dementia. Neuropsychologia, 38, 1207-1215.

Breedin, S. D., Saffran, E. M., \& Coslett, H. B. (1994). Reversal of the concreteness effect in a patient with semantic dementia. Cognitive Neuropsychology, 11, 617-660.

Bright, P., Moss, H., \& Tyler, L. K. (2004). Unitary vs. multiple semantics: PET studies of word and picture processing. Brain and Language, 89, 417-432.

Cardebat, D., Demonet, J. F., Celsis, P., \& Puel, M. (1996). Living/ nonliving dissociation in a case of semantic dementia: A SPECT activation study. Neuropsychologia, 34, 1175-1179.

Cipolotti, L., \& Warrington, E. K. (1995). Semantic memory and reading abilities: A case report. Journal of the International Neuropsychological Society, 1, 104-110.

Coccia, M., Bartolini, M., Luzzi, S., Provinciali, L., \& Lambon Ralph, M. A. (2004). Semantic memory is an amodal, dynamic system: Evidence from the interaction of naming and object use in semantic dementia. Cognitive Neuropsychology, 21, 513-527.

Coltheart, M. (1980). Deep dyslexia: A review of the syndrome. In M. Coltheart, K. Patterson, \& J. C. Marshall (Eds.), Deep dyslexia. London: Routledge and Kegan Paul.
Coltheart, M. (1981). The MRC Psycholinguistic Database. Quarterly Journal of Experimental Psychology, 33A, 497-505.

Coughlan, A. K., \& Warrington, E. K. (1981). The impairment of verbal semantic memory: A single case study. Journal of Neurology, Neurosurgery and Psychiatry, 44, 1079-1083.

Crinion, J. T., Lambon Ralph, M. A., Warburton, E. A., Howard, D., \& Wise, R. J. S. (2003). Temporal lobe regions engaged during normal speech comprehension. Brain, 126, 1193-1201.

Crutch, S. J., \& Warrington, E. K. (2006). Partial knowledge of abstract words in patients with cortical degenerative conditions. Neuropsychology, 20, 482-489.

D’Esposito, M., Detre, J. A., Aguirre, G. K., Stallcup, M., Alsop, D. C., Tippett, L. J., et al. (1997). A functional MRI study of mental image generation. Neuropsychologia, 35, 725-730.

Damasio, A. R. (1989). The brain binds entities and events by multiregional activation from convergence zones. Neural Computation, 1, 123-132.

Damasio, H., Tranel, D., Grabowski, T., Adolphs, R., \& Damasio, A. (2004). Neural systems behind word and concept retrieval. Cognition, 92, 179-229.

Davis, M. H., \& Johnsrude, I. S. (2003). Hierarchical processing in spoken language comprehension. Journal of Neuroscience, 23, 3423-3431.

DeGroot, A. M. B. (1989). Representational aspects of word imageability and word frequency as assessed through word association. Journal of Experimental Psychology: Learning, Memory, and Cognition, 15, 824-845.

Fiebach, C. J., \& Friederici, A. D. (2003). Processing concrete words: fMRI evidence against a specific right-hemisphere involvement. Neuropsychologia, 42, 62-70.

Funnell, E. (1995). Objects and properties: A study of the breakdown of semantic memory. Memory, 3, 497-518.

Giesbrecht, B., Camblin, C. C., \& Swaab, T. Y. (2004). Separable effects of semantic priming and imageability on word processing in human cortex. Cerebral Cortex, 14, 521-529.

Gloor, P. (1997). The temporal lobe and the limbic system. Oxford: Oxford University Press.

Goodglass, H., Hyde, M. R., \& Blumstein, S. (1969). Frequency, picturability and availability of nouns in aphasia. Cortex, 5, 104-119.

Grossman, M., \& Ash, S. (2004). Primary Progressive Aphasia: A review. Neurocase, 10, 3-18.

Grossman, M., Koenig, P., DeVita, C., Glosser, G., Alsop, D., Detre, J., et al. (2002). The neural basis for category-specific knowledge: An fMRI study. NeuroImage, 15, 936-948.

Hodges, J. R., Patterson, K., Oxbury, S., \& Funnell, E. (1992). Semantic dementia: Progressive fluent aphasia with temporal-lobe atrophy. Brain, 115, 1783-1806.

Howard, D., \& Patterson, K. (1992). Pyramids and palm trees: A test of semantic access from pictures and words. Bury St. Edmunds, Suffolk: Thames Valley Test Company.

James, C. T. (1975). The role of semantic information in lexical decisions. Journal of Experimental Psychology, 104, 130-136.

Jefferies, E., Baker, S. S., Doran, M., \& Lambon Ralph, M. A. (2007). Refractory effects in stroke aphasia: A consequence of poor semantic control. Neuropsychologia, 45, 1065-1079.

Jessen, F., Heun, R., Erb, M., Granath, D-O., Klose, U., Papassotiropoulos, A., \& Grodd, W. (2000). The concreteness effect: Evidence for dual coding and context availability. Brain and Language, 74, 103-112.

Jones, G. V. (1985). Deep dyslexia, imageability, and ease of predication. Brain and Language, 24, 1-19.

Kellenbach, M. L., Hovius, M., \& Patterson, K. (2005). A PET study of visual and semantic knowledge about objects. Cortex, 41, 121-132.

Kiehl, K., Liddle, P. F., Smith, A. M., Mendrek, A., Forster, B. B., \& Hare, R. D. (1999). Neural pathways involved in the processing of concrete and abstract words. Human Brain Mapping, 7, 225-233.

Kroll, J. F., \& Merves, J. S. (1986). Lexical access for concrete and abstract words. Journal of Experimental Psychology, 12, 92-107. 
Lambon Ralph, M. A., Graham, K. S., Ellis, A. W., \& Hodges, J. R. (1998). Naming in semantic dementia: What matters? Neuropsychologia, 36, 775-784.

Lambon Ralph, M. A., Graham, K. S., Patterson, K., \& Hodges, J. R. (1999). Is a picture worth a thousand words? Evidence from concept definitions by patients with semantic dementia. Brain and Language, 70, 309-335.

Lambon Ralph, M. A., Patterson, K., Garrard, P., \& Hodges, J. R. (2003). Semantic dementia with category specificity: A comparative case-series study. Cognitive Neuropsychology, 20, 307-326.

Luzzi, S., Snowden, J. S., Neary, D., Coccia, M., Provinciali, L., \& Lambon Ralph, M. A. (2007). Distinct patterns of olfactory impairment in Alzheimer's disease, semantic dementia, frontotemporal dementia, and corticobasal degeneration. Neuropsychologia, 45, 1823-1831.

Marshall, J., Pring, T., Chiat, S., \& Robson, J. (1996). Calling a salad a federation: An investigation of semantic jargon. Pt. 1: Nouns. Journal of Neurolinguistics, 9, 237-250.

McCarthy, R. A., \& Warrington, E. K. (1988). Evidence for modalityspecific meaning in the brain. Nature, 334, 428-430.

Mummery, C. J., Patterson, K., Price, C. J., Ashburner, J., Frackowiak, R. S. J., \& Hodges, J. R. (2000). A voxel-based morphometry study of semantic dementia: Relationship between temporal lobe atrophy and semantic memory. Annals of Neurology, 47, 36-45.

Narain, C., Scott, S. K., Wise, R. J. S., Rosen, S., Leff, A., Iversen, S. D., et al. (2003). Defining a left-lateralized response specific to intelligible speech using fMRI. Cerebral Cortex, 13, 1362-1368.

Nestor, P. J., Fryer, T. D., \& Hodges, J. R. (2006). Declarative memory impairments in Alzheimer's disease and semantic dementia. NeuroImage, 30, 1010-1020.

Noppeney, U., \& Price, C. J. (2002). Retrieval of visual, auditory and abstract semantics. NeuroImage, 15, 917-926.

Noppeney, U., \& Price, C. J. (2004). Retrieval of abstract semantics. NeuroImage, 22, 164-170.

Paivio, A. (1986). Mental representations: A dual coding approach. Oxford: Oxford University Press.

Paivio, A. (1991). Dual coding theory: Retrospect and current status. Canadian Journal of Psychology, 45, 255-287.

Papagno, C., Capasso, R., Zerboni, H., \& Miceli, G. (2007). A reverse concreteness effect in a subject with semantic dementia. Brain and Language, 103, 90-91.

Patterson, K., Lambon Ralph, M. A., Jefferies, E., Woolams, A., Jones, R., Hodges, J., et al. (2006). 'Pre-semantic' cognition in Semantic Dementia: Six deficits in search of an explanation. Journal of Cognitive Neuroscience, 18, 169-183.

Perani, D., Schnur, T., Tettamanti, C., Gorno-Tempini, M., Cappa, S. F., \& Fazio, F. (1999). Word and picture matching: A PET study of semantic category effects. Neuropsychologia, 37, 293-306.

Plaut, D. C., \& Shallice, T. (1993). Deep dyslexia: A case-study of connectionist neuropsychology. Cognitive Neuropsychology, 10, 377-500.

Pobric, G., Lambon Ralph, M. A., \& Jefferies, E. (in press). The role of the anterior temporal lobes in the comprehension of concrete and abstract words: rTMS evidence. Cortex.

Pulvermüller, F., Pye, E., Dine, C., Hauk, O., Nestor, P., \& Patterson, K. (2008). Word category deficits in semantic dementia. Paper presented at the Cognitive Neuroscience Society 2008 Annual Meeting.

Raven, J. C. (1962). Coloured progressive matrices sets A, AB, B. London: H. K. Lewis.

Reilly, J., Cross, K., Troiani, V., \& Grossman, M. (2007a). Single-word semantic judgements in semantic dementia: Do phonology and grammatical class count? Aphasiology, 21, 558-569.

Reilly, J., Grossman, M., \& McCawley, M. C. (2006). Concreteness effects in lexical processing of semantic dementia. Brain and Language, 99, $157-158$.
Reilly, J., Peelle, J. E., \& Grossman, M. (2007b). A unitary semantics account of reverse concreteness effects in semantic dementia. Brain and Language, 103, 86-87.

Rogers, T. T., Lambon Ralph, M. A., Garrard, P., Bozeat, S., McClelland, J. L., Hodges, J. R., et al. (2004). The structure and deterioration of semantic memory: A neuropsychological and computational investigation. Psychological Review, 111, 205-235.

Rogers, T. T., \& McClelland, J. L. (2004). Semantic cognition: A parallel distributed processing approach. Cambridge, MA: MIT Press.

Sabsevitz, D. S., Medler, D. A., Seidenberg, M., \& Binder, J. R. (2005). Modulation of the semantic system by word imageability. NeuroImage, 27, 188-200.

Schwanenflugel, P. J., \& Shoben, E. J. (1983). Differential context effects in the comprehension of abstract and concrete verbal materials. Journal of Experimental Psychology: Learning, Memory, and Cognition, 9, 82-102.

Scott, S. K., Blank, S. C., Rosen, S., \& Wise, R. J. S. (2000). Identification of a pathway for intelligible speech in the left temporal lobe. Brain, 123, $2400-2406$.

Sirigu, A., Duhamel, J. R., \& Poncet, M. (1991). The role of sensorimotor experience in object recognition: A case of multimodal agnosia. Brain, 114, 2555-2573.

Snowden, J. S., Goulding, P. J., \& Neary, D. (1989). Semantic dementia: A form of circumscribed cerebral atrophy. Behavioural Neurology, 2, $167-182$.

Stewart, L., Meyer, B. U., Frith, U., \& Rothwell, U. (2001). Left posterior BA37 is involved in object recognition: A TMS study. Neuropsychologia, 39, 1-6.

Ungerleider, L. G., \& Mishkin, M. (1982). Two cortical visual systems. In D. J. Ingle, M. A. Goodale, \& R. J. W. Mansfield (Eds.), Analysis of visual behaviour. Cambridge, MA: MIT Press.

Vandenberghe, R., Price, C., Wise, R., Josephs, O., \& Frackowiak, R. S. J. (1996). Functional anatomy of a common semantic system for words and pictures. Nature, 383, 254-256.

Vesely, L., Bonner, M. F., Reilly, J., \& Grossman, M. (2007). Free association in semantic dementia: The importance of being abstract. Brain and Language, 103, 154-155.

Warrington, E. K. (1975). Selective impairment of semantic memory. Quarterly Journal of Experimental Psychology, 27, 635-657.

Warrington, E. K., \& James, M. (1991). The visual object and space perception battery. Bury St. Edmunds, Suffolk: Thames Valley Test Company.

Wechsler, D. (1987). Wechsler Memory Scale - Revised (WMS-R). New York: Psychological Corporation.

Whatmough, C., Verret, L., Fung, D., \& Chertkow, H. (2004). Common and contrasting areas of activation for abstract and concrete concepts: $\mathrm{An}_{2}{ }^{15} \mathrm{O}$ PET study. Journal of Cognitive Neuroscience, 16, 1211-1226.

Wise, R. J. S., Howard, D., Mummery, C. J., Fletcher, P., Leff, A., Buchel, C., et al. (2000). Noun imageability and the temporal lobes. Neuropsychologia, 38, 985-994.

Woollams, A. M., Lambon Ralph, M. A., Plaut, D. C., \& Patterson, K. (2007). SD-squared: On the association between semantic dementia and surface dyslexia. Psychological Review, 114, 316-339.

Yi, H. A., Moore, P., \& Grossman, M. (2007). Reversal of the concreteness effect for verbs in patients with semantic dementia. Neuropsychology, 21, 9-19.

Received April 30, 2008

Revision received November 7, 2008 Accepted November 11, 2008 